\title{
Prekallikrein and high molecular weight kininogen deficiency in Oman: a challenging diagnosis in mucosal bleeding
}

\begin{abstract}
Background: Prekallikrein (PK) and high molecular weight kininogen (HMWK) are contact factors that are involved in the intrinsic pathway of the coagulation cascade. Deficiency of PK or HMWK are known to be associated with prolonged a PTT, but no clinical bleeding. However, rare cases of PK deficiency have been reported to be associated with mucosal bleeding.
\end{abstract}

Objective: To report on epidemiological and clinical characteristics of Omani patients with PK and HMWK deficiency, focusing on one symptomatic case.

Patients and methods: We reviewed the files of Omani patients who had persistently prolonged a PTT that proved to be secondary to PK or HMWK deficiency over a period of ten years.

Results: Eight cases (three with PK, five with HMWK deficiency) were identified. All but one case were asymptomatic. A thirteen year-old female with Hashimoto thyroiditis who presented with easy bruising, severe prepubertal vaginal bleeding and recurrent hematemesis proved to have prekallikrein deficiency. Acquired vWD, coagulation factors deficiency, lupus anticoagulant, platelet dysfunction were ruled out. No local cause of bleeding could be identified even after four endoscopic examinations, Meckel's diverticulum scintigraphy and CT angiography of the abdomen.

Conclusion: PK and HMWK are underestimated in Oman. Most cases were incidentally detected. They significantly impact medical costs, related to extensive laboratory testing and undue delay in planned surgical procedures. Some cases with clinical bleeding impose a diagnostic challenge.

Keywords: contact factors deficiency, high molecular weight kininogen, oman, prekallikrein, prolonged aPTT
Volume 7 Issue I - 2019

\author{
Hanan F Nazir, ${ }^{1,2}$ AnilV Pathare ${ }^{3}$ \\ 'Child Health Department, Sultan Qaboos University Hospital, \\ Muscat, Oman \\ ${ }^{2}$ Department of Pediatrics, Alexandria Faculty of Medicine, \\ Alexandria, Egypt \\ ${ }^{3}$ Department of Haematology, Sultan Qaboos University, Muscat, \\ Oman
}

Correspondence: Hanan Fawzy Nazir, Child Health Department, SQUH, Alkhoudh, Muscat I23, PO Box 38, Oman, Tel +96824 I44II0; +96896556198, Fax +968 24I4II36, Email dr.hanannazir@yahoo.com

Received: January 29, 2019 | Published: February 27, 2019
Abbreviations: PK, Prekallikrein; HMWK, High Molecular Weight Kininogen; vWD, Von Willebrand Disease; OGD, Oesophago-gastroduodenoscopy; CLO test, Campylobacter-like organism test(for H Pylori; BPH, Benign prostatic hyperplasia; CVA, Cerebrovascular accident; AF, Atrial fibrillation; CKD, Chronic kidney disease

\section{Introduction}

The contact system includes FXII, FXI, prekallikrein (PK), and high molecular weight kininogen (HMWK). This system is activated in the presence of negatively charged surface, leading to activation of FXI, followed by a chain of proteolytic reactions, resulting ultimately in thrombin generation and fibrin clot formation.

Lack of clinical bleeding in cases of contact factors deficiency is supported by several reports, in which abnormally prolonged aPTT was accidentally revealed on screening tests. Bypassing the role of $\mathrm{PK}$ and other contact activation system can take place in vivo through different mechanisms. First, activated platelets can trigger FXI activation, independent of FXIIa. Second, tissue factor that is exposed by endothelial damage, can bind to FVIIa, leading to activation of FIX and FX. As a result, thrombin is generated, which causes fibrin and FXIa formation. In addition, long chain polyphosphate can activate the intrinsic pathway independently from the contact factors. These mechanisms explain the lack of clinical bleeding in most patients with PK and other contact factors deficiency. On the other hand, clinical bleeding has been rarely reported to be associated with PK deficiency. ${ }^{1}$
The objective of the current work is to report on epidemiological and clinical characteristics of Omani patients with PK and HMWK deficiency, focusing on abnormal bleeding in one child with PK deficiency.

\section{Subjects and methods}

We reviewed the files of patients investigated in Sultan Qaboos University Hospital (SQUH) between 2000- 2018, for prolonged aPTT and were confirmed to have PK or HMWK deficiency. Data was collected regarding their epidemiological characteristics, indication to investigate for coagulation screening, screening laboratory results, clinical phenotype and impact on their management. Assays for PK and HMWK were based on commercially available factor deficient Fletcher's and Fitzgerald's trait plasma. This report focuses on the details of a child with PK deficiency who had significant mucosal bleeding.

\section{Results}

Between 1999-2018, a total of eight patients were identified; two males $(25 \%)$, and six females $(75 \%)$ from unrelated kind reds. Three patients had PK deficiency; five patients had HMWK deficiency, in addition to more than 1000 patients who had factor XII deficiency (not included in the report). Their age at diagnosis ranged between 2-80 years (mean \pm SD: $26.3 \pm 26.7$ ). Indications for coagulation screening were preoperative assessment in five patients $(62.5 \%)$, bleeding in two patients $(25 \%)$ and family screening in one patient $(12.5 \%)$. Median time from screening test to definitive diagnosis ranged from 3 to 8 
months. All but one patient had no history of abnormal bleeding. They required no special preparation before surgery and post-operative course was unremarkable.

Table 1 summarizes the epidemiological, clinical, and laboratory data of patients with PK or HMWK deficiency. A 13 year-old child with PK deficiency and a background diagnosis of Hashimoto thyroid it is presented with significant abnormal bleeding. Hypothyroidism was diagnosed at the age of 7 years, was treated with L- thyroxin, with poor compliance.

Table I Demographic clinical and laboratory data of patients with PK or HMWK deficiency

\begin{tabular}{|c|c|c|c|c|c|c|c|}
\hline & 1 & 2 & 3 & 4 & 5 & 6 & 7 \\
\hline Sex & $\mathrm{F}$ & $\mathrm{F}$ & $\mathrm{M}$ & $\mathrm{F}$ & M & $\mathrm{F}$ & $\mathrm{F}$ \\
\hline $\begin{array}{l}\text { Age in years } \\
\text { (current/age at diagnosis) }\end{array}$ & $14 / 5$ & $9 / 2$ & $17 / 11$ & $49 / 48$ & $84 / 80$ & $37 / 34$ & $21 / 21$ \\
\hline Diagnosis & HMWK & HMWK & HMWK & HMWK & PK & PK & HMWK \\
\hline PTsec & 10.5 & 10.4 & 11 & 10.6 & 11.2 & 10.5 & 10.7 \\
\hline INR & 0.98 & 0.95 & 1.1 & 1.02 & 1 & 0.96 & 0.99 \\
\hline aPTT sec & $>180$ & $>180$ & $>180$ & $>180$ & $>180$ & 167.9 & $>180$ \\
\hline aPTT mixing sec & 41 & 41 & 40 & 38.4 & 39.6 & 33.6 & 33.6 \\
\hline Fibrinogen $\mathrm{g} / \mathrm{L}$ & 1.8 & 2.1 & 2.5 & 3.1 & 4.5 & 4.7 & 3.5 \\
\hline TT sec & 14.9 & 15 & 14 & 14.5 & 14.8 & 19.5 & 16 \\
\hline PK (0.650-1.300)iu/ml & 0.507 & 0.510 & $\begin{array}{l}0.456 \\
(0.530-1.450)\end{array}$ & 0.550 & 0.002 & 0.062 & 0.516 \\
\hline HMWK (0.640-1.320)iu/ml & 0.001 & 0.008 & $<0.001$ & $<0.007$ & $\begin{array}{l}0.311 \\
(0.500-1.360)\end{array}$ & 0.884 & $<0.007$ \\
\hline F VIII 0.495-1.382 iu/ml & 0.493 & 0.770 & 1.030 & 0.760 & 2.206 & 1.422 & 1.180 \\
\hline FIX 0.470-1.040iu/ml & 0.564 & 0.825 & 0.940 & 0.846 & 1.737 & 1.102 & 1.100 \\
\hline FXI $0.560-1.500 \mathrm{iu} / \mathrm{ml}$ & 0.558 & 0.554 & 0.568 & 0.580 & 0.565 & 0.563 & 0.570 \\
\hline FXII 0.640-1.290 iu/ml & 0.640 & 0.840 & 0.780 & 1.220 & 0.747 & 1.315 & 0.870 \\
\hline vWD assay & Normal & Normal & Normal & Normal & Normal & Normal & Normal \\
\hline Bleeding & Bloody diarrhea & None & None & None & None & Bleeding gums & None \\
\hline Transfusion & FFP & Non & None & None & None & None & None \\
\hline Family history & + ve & $+\mathrm{ve}$ & -ve & -ve & -ve & -ve & -ve \\
\hline Comorbidity & Bloody diarrhea & OM & None & None & $\begin{array}{l}\text { CVA } \\
\text { BPH post open } \\
\text { prostatectomy } \\
\text { IHD/AF } \\
\text { CKD }\end{array}$ & None & None \\
\hline Comments & $\begin{array}{l}\text { No follow up } \\
\text { since } 2012\end{array}$ & $\begin{array}{l}\text { No follow up } \\
\text { since } 2011\end{array}$ & - & - & $\begin{array}{l}\text { Multiple surgical } \\
\text { procedures without } \\
\text { bleeding }\end{array}$ & $\begin{array}{l}4 \text { SVD, no } \\
\text { bleeding }\end{array}$ & - \\
\hline
\end{tabular}

Abbreviations: PK, prekallikrein; HMWK, high molecular weight kininogen; VWD, Von Willebrand disease; FFP, fresh frozen plasma; OM, otitis media; CVA cerebrovascular accident; BPH, benign prostatic hyperplasia; IHD, ischemic heart disease; AF, atrial fibrillation; CKD, chronic kidney disease; SVD, spontaneous vaginal delivery

At the age of ten years, she was referred from her primary hospital to SQUH for further evaluation of a persistently prolonged aPTT with recurrent bleeding. She gave history of easy bruising and prolonged vaginal bleeding that lasted for three weeks, in addition to four episodes of hematemesis over a period of six months. There was no history of epistaxis, hematuria, hemarthrosis, or other complaints. Her parents are non-consanguineous and family history was negative for bleeding tendency. On arrival to the hematology outpatient clinic, she collapsed with hypovolemic shock and was immediately resuscitated with normal saline boluses and shifted to PICU, where she received packed red blood (pRBCs) and fresh frozen plasma transfusion (FFP). Clinical examination revealed a well oriented adolescent girl, with marked pallor and no icterus. She was afebrile, blood pressure 87/49 $\mathrm{mmHg}$, heart rate 110 beats/min, capillary refill time (CRT) of $4 \mathrm{sec}$, and her peripheral pulses were feeble. Cardiovascular examination revealed tachycardia with normal heart sounds and no gallop rhythm. Chest examination was unremarkable, her abdomen was soft, with no focal tenderness and no organomegaly. There was no ecchymosis, petechial rash or telangiectasia on her skin or oral mucosa, and her CNS examination showed no focal neurological deficits. Anthropometric measurement showed that her weight was between 50th-75th centile and height at 75 th centile for age. $X$ ray wrist showed her bone age to be between $9-10$ years.

Clinical presentation and laboratory tests at different admission episodes are summarized in Table 2. Laboratory tests were marked for microcytic hypochromic anemia, normal platelet, normal total and differential WBCs counts. Iron profile showed iron deficiency. Renal function tests, serum electrolytes and liver function test results were within normal levels. Her TFT initially revealed hypothyroidism (T4:5.2, TSH $>100$ ) that later normalized on L-thyroxin replacement. Repeated coagulation screenings revealed normal PT, normal TT and normal fibrinogen level, with a markedly prolonged aPTT on several occasions (111.7-164.1sec). Mixing study using 1:1normal plasma completely corrected aPTT, suggesting of factor deficiency. 
Table 2 Clinical presentation and laboratory tests during different hospital admissions in a child with PK deficiency and abnormal bleeding

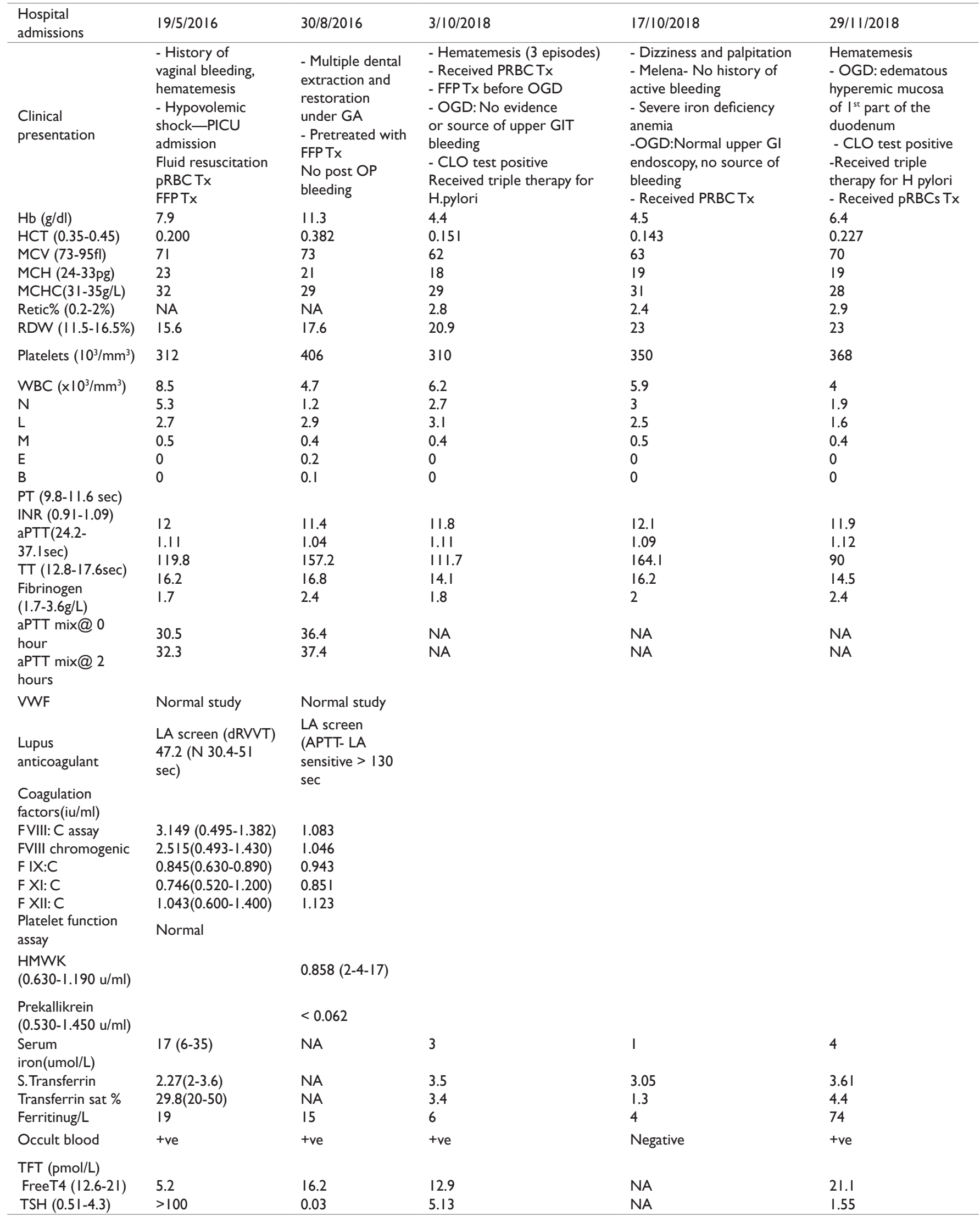

Abbreviations: PICU, pediatric intensive care unit; pRBCTx, packed red blood cell transfusion; FFP,Tx, fresh-frozen plasma transfusion; GA, general anaesthesia; OGD, oesophago- gastroduodenoscopy; CLO test, campylobacter-like organism test (for H pylori) 
In view of her neglected hypothyroidism, next step in her evaluation was to rule out acquired VWD and other coagulation factor deficiencies that are related to deficient production. The presence of lupus anticoagulant was also thought of, considering her primary diagnosis of Hashimoto thyroiditis. Surprisingly, her laboratory test results ruled out all of the speculated abnormalities. VWD workup was normal twice and coagulation factors assay were normal. Lupus anticoagulant was negative, ruling it out as a possible cause of prolonged aPTT. Thus, the next step was to test for HMWK and PK level. HMWK level was normal $0.858 \mathrm{iu} / \mathrm{ml}$ (RR 0.630-1.190), while PK level was severely deficient $(<0.062 \mathrm{iu} / \mathrm{ml}$ (RR $0.530-1.450)$.

On follow up over a period of three years, her clinical course was marked with four hospital admissions: three times for management of severe anemia related to hematemesis and melena, and once for dental extraction and restoration under general anaesthesia. Diagnostic workup for GIT bleeding included upper and lower endoscopic examination three times, that have been reported as normal, with no evidence of gastric/duodenal ulceration, or any evident source of bleeding. Meckel's diverticulum scintigraphy using (Tc99m$125 \mathrm{MBq}$ ) showed normal physiological radiotracer uptake, ruling out a local cause of her recurrent mucosal bleeding. CT angiography of the abdomen was requested to look for a source of GIT bleeding, and was reported as normal. Before establishing the diagnosis of PK deficiency, she received FFP transfusion before dental extraction that resulted in normalization of APTT and no post-operative bleeding.

\section{Discussion}

PK and HMWK deficiency are rare causes of persistently prolonged aPTT. In Oman, these disorders were identified in eight cases over a period of nine years. However, the true prevalence may be under estimated, in view of the asymptomatic nature of the defect and the fact that these patients were referred for evaluation. Most cases were detected incidentally, on preoperative screening. Although these conditions are not associated with significant bleeding, still they significantly impact clinical management of patients, in terms of costs of extensive investigation, and undue delay of surgical procedures. In Oman, the average cost of extensive laboratory testing for coagulation factors assay is 75USD/assay and vWD workup is around 150USD. Furthermore, in the reported cases, there was a significant delay in planned surgical procedures between 3-6 months.

On the other hand, in the current report, the child who had significant bleeding imposed a diagnostic challenge. She was diagnosed with Hashimoto thyroiditis since the age of seven years. On presentation, her hypothyroid status was poorly controlled, as evidenced by low free T4, and very high TSH levels. Before reaching the diagnosis of PK deficiency, it was more plausible to rule out acquired vWD and other coagulation factor deficiency that are known to be associated with hypothyroidism.

Acquired vWD type 1 seems to be the most common reason behind abnormal bleeding in patients with hypothyroidism. In those cases, low levels of VWF are related to reduction in its synthesis and/or secretion. Moreover, acquired vWD in cases of Hashimoto thyroiditis is reported independently of thyroid hormone levels, most likely related to the rapid clearance of FVIII/VWF complex due to binding of non-neutralizing autoantibodies to $\mathrm{VWF}^{2}$

Prepubertal vaginal bleeding in the current case could be viewed in 2 different ways: either as a manifestation of generalized coagulopathy or as a manifestation of precocious puberty related to her hypothyroidism. Increased TSH levels in primary hypothyroidism can stimulate FSH receptors because of molecular similarity between these two hormones (specificity spill over), leading to precocious puberty. ${ }^{3}$ This condition is reversible upon replacement therapy with thyroid hormones. It is mostly encountered in cases of prolonged hypothyroidism, which is usually reflected also on deceleration of linear growth, a finding that was lacking in the current case (height at 75th centile, and appropriate bone age). On the other hand, no more vaginal bleeding was seen after normalization of her hypothyroid state, despite her persistently prolonged aPTT, arguing more in favor of being related to hypothyroidism.

In addition, the diagnosis of autoimmune thyroid disorder (Hashimoto thyroiditis), provoked the need to rule out other autoimmune conditions that could be associated. ${ }^{4}$ Idiopathic thrombocytopenic purpura, secondary antiphospholipid syndrome, and acquired hemophilia have been ruled out in the current case, based on her normal platelet count, negative antiphospholipid antibodies, and normal coagulation factors assay.

Progressively severe iron deficiency was notable in the current case and it is related most probably to her acute and chronic blood loss. The effect of iron deficiency anemia on hemostasis is double- faceted. Anemia has been observed in vivo to produce a hypercoagulable state through acceleration of platelet aggregation. Also, iron deficiency may promote thrombosis via elevation of plasma level of FVIII. On the other hand, anemia is associated with a delay in the initiation of the coagulation cascade, leading to prolonged bleeding time, but improves viscoelastic properties and strength of the clot, and had no effect on coagulation time. ${ }^{5,6}$ As the patient did not tolerate oral iron, she was treated with intravenous ferrous sulfate infusion. Serum iron before replacement was 1 umol/L (Normal: 6-35), ferritin was $4 \mathrm{ug} / \mathrm{L}$ (Normal:7-84), and transferrin saturation was 3.5\% (normal 20-50\%). On discharge, a different oral iron preparation (ferrous sulfate syrup) was prescribed and she was encouraged on compliance. However, in view of repeated losses, her iron deficiency was difficult to correct.

Although severe PK deficiency is known to cause prolonged aPTT, it is rarely reported to be associated with clinical bleeding. Severe PK deficiency is a rare disorder, till 2017, almost 150 cases have been reported. (1)Rarely, clinical bleeding has been reported by some authors to be associated with PK deficiency. In 2017, Antonio G and coauthors, ${ }^{7}$ reviewed 106 papers on PK deficiency, and critically analyzed the few case reports that claimed an association with significant bleeding diathesis. Among 100 cases with PK deficiency, ten cases were reported to have clinical bleeding, representing $10 \%$, similar to bleeding cases in the current report (one out of eight; $12.5 \%$ ). Reported bleeding varied between mild (ecchymosis, epistaxis, oral mucosal bleeding after surgery) to severe (presenting with hemarthrosis and requiring transfusion of $\mathrm{pRBCs}$, or FFP). He argued that most of the reported cases had undergone surgical procedures (like tonsillectomy, circumcision, delivery, and even hysterectomy) with no significant bleeding, thus excluding a bleeding tendency. He suggested that local factors or transient coagulation defects are the likely explanation for the occasional bleeding manifestations observed in those cases. In the current case, a local factor for recurrent hematemesis and melena could not be found, even after three endoscopic examinations, Mickel's scan and CT angiography of the abdomen. Transient coagulation defects, like acquired $\mathrm{vWD}$, or other coagulation factors deficiency related to her thyroid disorder were also ruled out.

In few cases with PK deficiency, significant bleeding that responded to FFP transfusion was probably attributed toPK deficiency. A specific mutation that led to a bleeding phenotype was proposed as 
a possible explanation. Nevertheless, molecular genetic testing have been applied to few families so far, and these studies do not yet allow definite phenotype/genotype correlations. ${ }^{8,9}$

The current case represented a diagnostic challenge, in view of the multiple sites of bleeding, severity of resulting anemia requiring multiple transfusions, and no obvious local cause to explain bleeding. Whether bleeding in the current case is related to PK deficiency is still unknown and might be added to previously reported symptomatic PK deficiency cases. Unfortunately, specific genetic testing for the exact mutation was not available in our laboratory.

\section{Conclusion}

PK and HMWK are rare and most certainly underestimated in Oman. Most cases were detected upon investigations for prolonged aPTTon pre-operative screening. However, they have asignificant impact on costs related to extensive laboratory testing and delay of planned surgical procedure. Additionally, some cases with clinical bleeding impose a diagnostic challenge. Further studies to explore possible genotype-phenotype relation are needed.

\section{Conflict of interest and sources of funding}

Funding source: Authors have no financial relations relevant to this article to disclose.

Conflict of interests: Authors have no conflict of interests relevant to this article to disclose.

\section{Acknowledgments}

None.

\section{References}

1. Zheng S, Just S, Brighton T. Prekallikrein deficiency. Pathology. 2016;48(6):634-637.

2. Koyama T, Fujimoto K, Shima M. Acquired von Willebrand syndrome associated with Hashimoto's thyroiditis and subcutaneous mucosaassociated lymphoid tissue lymphoma. Intern Med. 2013;52(23):26612663.

3. Ghaemi N, Vakili R, Bagheri S. Precocious Puberty: An Unusual Presentation of Hypothyroidism. International Journal of Pediatrics. 2013;1(2):51-54.

4. Squizzato A, Romualdi E, Büller HR, et al. Thyroid dysfunction and effects on coagulation and fibrinolysis: a systematic review. $J$ Clin Endocrinol Metab. 2007; 92(7):2415-2420.

5. Roeloffzen WW, Kluin-Nelemans HC, Bosman L, et al. Effects of red blood cells on hemostasis. Transfusion. 2010;50(7):1536-1544.

6. Livesey JA, Manning RA, Meek JH, et al. Low serum iron levels are associated with elevated plasma levels of coagulation factor VIII and pulmonary emboli/deep venous thromboses in replicate cohorts of patients with hereditary haemorrhagic telangiectasia. Thorax. 2012;67(4):328-333.

7. Girolami A, Cosi E, Ferrari S, et al. Critical Analysis of the Patients with Congenital Prekallikrein Deficiency and a Purported Bleeding Tendency. J Mol Genet Med. 2017(1)1:9

8. Girolami A, Scarparo P, Candeo N, et al. Congenital prekallikrein deficiency. Expert Review of Hematology. 2010;3(6):685-695.

9. Girolami A, Ferrari S, Cosi E, et al. A structure-function analysis in patients with prekallikrein deficiency. Hematology. 2018;23(6):346350 . 